\section{Structural models programs: NOVAL and BALANCE*}

\author{
RONALD G. SHERWIN $\dagger$ \\ Department of International Relations \\ University of Southern California \\ Los Angeles, California 90007 \\ and

\section{GARY KLEIN} \\ Jet Propulsion Laboratories \\ Pasadena, California 91100
}

One widely accepted principle in the behav $d$ sciences is that the structure of relations within a oup is an important factor for determining the future ate of the group's relations. Stemming from this principle is the general notion that affective linkages in triadic groups will usually assume a balanced configuration. Collectively, the theories which de with the structure of affective relations in triads are, ed balance theory. Balance theory and other structura heories of behavior have been discussed extensively $i_{\text {. }}$ the social science literature. ${ }^{1}$

Paralleling the theoretical development of generalized structural theories of behavior has been the development of systematic techniques for deciphering the properties of interaction structures. The bulk of this work is summarized by Harary, Norman, and Cartwright (1965) and Harary (1969). In addition to the more general mathematical work concerning interaction structures, there have appeared several techniques for rendering affective relations amenable to systematic analysis. Not until recently, however, has the problem of systematically describing affective interaction structures in relatively large groups been solved to the point where computers may be used to test models and hypotheses derived from theories of balance. ${ }^{2}$ The solution to this problem is embodied in two computer programs: NOVAL and BALANCE.

Computer and Language. NOVAL and BALANCE are companion programs written in FORTRAN and currently operating in an IBM 360-370 environment. Briefly, NOVAL embodies principles for discovering a groups nonvalenced structures, while BALANCE focuses on valenced, or affective, interaction structures.

\section{NOVAL}

Input. Input to NOVAL is from a user-supplied adjacency matrix whose cells contain either zeros or

*This research was supported by ONR-ARPA Contract No. N00014-67-A-0296-0004.

+ Now at the Institute of International Studies, University of California, Berkeley. positive integers. The adjacency matrix may be as large as $20 \times 20$.

NOVAL analyzes dyadic and triadic relations without reference to any particular theoretical concept. Thus, the data in a NOVAL input matrix can be relevant to any of a large number of interaction variables, e.g., group interaction flows, international trade flows, mail flows, "responsiveness" measures, and so forth. Exactly what the data in a NOVAL data matrix signify is left to $t$ - user's discretion.

JVAL produces information concerning paths, cles, and semicycles in dyadic and triadic structures. NOVAL will also perform several analyses on the same data matrix. By manipulating the program's control parameters, several passes can be made through a data matrix with relationships at different thresholds of theoretical significance being considered during each pass. Similarly, NOVAL will print information about selected members of the original group, as well as information concerning the entire group.

Output. For every pass through the data matrix, NOVAL prints a list of nonvalenced triadic cycles plus a list of nonvalenced triadic semicycles. The cycles list has the following format: Unsigned Cycles

$\begin{array}{ccc}651 & 652 & 645 \\ . & \cdot & \cdot \\ . & . & . \\ . & . & .\end{array}$

This listing is fairly self-explanatory. It means that the linkages among 651,652 , and 645 , which fall within the user-specified thresholds, have the following configuration.

The semicycles list has the following format:

\begin{tabular}{ccc} 
& \multicolumn{2}{c}{ Unsigned Semicycles } \\
Sender-Sender & Sender-Receiver & Receiver-Receiver \\
002 & 651 & 666 \\
$\cdot$ & $\cdot$ & $\cdot$ \\
$\cdot$ & $\cdot$ & $\cdot$ \\
$\cdot$ & $\cdot$ & $\cdot$
\end{tabular}

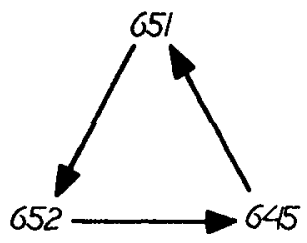

Fig. 1. Triadic cycle. 


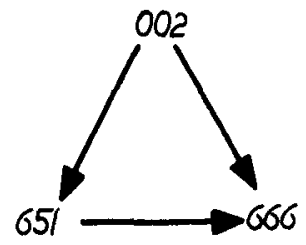

Fig. 2. Triadic semicycle.

It means that the relations among entities 002,651 , and 666 have the configuration shown in Fig. 2.

For each pass through the matrix, NOVAL also prints the following structural matrices: $\mathrm{A}, \mathrm{A}^{2}$, and $\mathrm{A} \cap \mathrm{A}^{\prime}$.

\section{BALANCE}

While NOVAL is a utility program for analyzing general interaction structures, BALANCE is markedly different since it is designed specifically to analyze affective balance structures: BALANCE analyzes friend-foe, or positive-negative relations and casts them into a triadic balance framework.

Despite the major conceptual difference between the two programs, the procedures and user options required to run both programs are very much the same so that running BALANCE is not much different from running NOVAL. Most of the conventions and programming rules that apply to NOVAL also apply to BALANCE. Thus, the user may control the number of passes through his data, the data's upper and lower thresholds of significance, and which relations are to be singled out for special treatment.

Input. BALANCE requires two input matrices: an adjacency matrix depicting a group's positive set of relations, and a matrix depicting the groups's negative set of relations. The matrices are referred to as $\mathrm{A}(\mathrm{P})$ and $A(N)$, respectively.

Output. BALANCE produces two tables, "valenced cycles" and "valenced semicycles," which are similar to the tables produced by NOVAL. However, the cycles and semicycles contained in BALANCE output are further identified according to a taxonomy of structurally balanced and unbalanced triads. This taxonomy is described by Sherwin (1974). It is also available as part of the program documentation described below.

In addition to the tables, BALANCE produces the following structural matrices: $A(P), A(N), A(P)^{2}, A(N)^{2}$, $A(P) \times A(N)$, and $A(N) \times A(P)$. These matrices provide information concerning the group's dyadic structure, as well as information concerning the group's "incomplete" triads. The program also prints a series of intersection matrices which are required to determine the presence of the particular triadic valence structures within the group's net of affective relations.

Limitations. NOVAL and BALANCE are currently batch mode programs written in FORTRAN. They were tested on an IBM 370/155. NOVAL's largest core requirement was $108 \mathrm{~K}$, and it required $7.01 \mathrm{sec}$ to analyze a $9 \times 9$ matrix. The largest core requirement for BALANCE was $112 \mathrm{~K}$, and $17.56 \mathrm{sec}$ were required to analyze a $10 \times 10$ matrix.

Program Availability. Program listings and documentation are available from the International Relations Research Institute, Department of International Relations VKC-330, University of Southern California, Los Angeles, California 90007. The cost for listings and documentation of both NOVAL and BALANCE is $\$ 5$. Source decks for both programs may be obtained for an additional $\$ 15$.

\section{REFERENCES}

Abelson, R., et al (Eds.). Theories of cognitive consistency: $A$ sourcebook. Chicago: Rand McNally, 1968.

Harary, F., Norman, R., \& Cartwright, D. Structural models: An introduction to the theory of directed graphs. New York:

Harary, 1965 . $\widetilde{G}$. Addison-Wesley, 1969.

Sherwin, R. Structural balance and the sociomatrix: Finding triadic valence structures in signed adjacency matrices. Human Relations, 1974 , in press.

\section{NOTES}

1. The best single source on theories of balance and the issues concerning them is Abelson et al (1968).

2. A set of algorithms for processing a signed adjacency matrix in order to discern its underlying triadic structures has been developed by Sherwin (1974). Sherwin also cites other work concerning the mathematical properties of signed adjacency
matrices. 